\title{
Repositorio del Instituto Nacional de Pediatría
}

\author{
Repository of the National Pediatrics Institute
}

Desde su fundación, el Instituto Nacional de Pediatría se ha consolidado como un centro pionero en el ramo de la investigación y la enseñanza.

Estas dos plataformas estarían incompletas sin un centro de información documental de vanguardia. Desde 1977, la biblioteca de este instituto ha estado en constante renovación; primero, con la creación de sus instalaciones y la constante actualización de su acervo, además, destacar la obtención de recursos y materiales, como lectores de CD en 1988, y hasta la donación de equipos de computo 1999.

En el año 2012, se puso en marcha la página web del INP, en la que se da servicio remoto a todo el país. En esta plataforma, los pediatras y médicos generales de México y Centro y Sur América tienen acceso a libros y documentos electrónicos en cualquier parte del país. Aunado a este gran acervo (2,283 libros, 548 títulos de pediatría, 1,541 obras de consulta, 1,417 tesis, 177 títulos de archivo histórico y 675 títulos de publicaciones periódicas) con el que cuenta la biblioteca, en 2014 el CONACYT nos dio acceso a bases de datos de diversas universidades del país y extranjeras, así como a diversas publicaciones médicas en las que se puede consultar lo más reciente en investigación médica.

Este año el Instituto Nacional de Pediatría al contar con su Repositorio Institucional, se consolida como el primer Instituto Nacional de Salud con una plataforma digital en la que se preserva, almacena y archiva, de acuerdo con estándares internacionales, toda la producción de la investigación científica. Esta iniciativa la lanzó CONACYT en 2015, y forma parte de la implementación de política de acceso abierto y de ciencia abierta que busca que los productos de investigación, que son financiados parcial o totalmente con recursos públicos, y que estén disponibles en línea de forma gratuita para toda la sociedad. Este Repositorio institucional se sumará a uno nacional en el que la investigación médica alcanzará la consulta del $80 \%$ de la información médica de actualidad.

La conformación del Repositorio del INP tuvo sus inicios en 2015, año en el que se emitió la primera convocatoria por parte del CONACYT y en la que la Lic. Cecilia Solís Galicia y el Lic. José Luis Galindo, responsables del Departamento de 
Servicios Bibliotecarios del INP, se dieron a la tarea de desarrollar el proyecto que fue evaluado y aprobado en abril de 2016, junto con 36 instituciones más.

El trabajo para consolidar el repositorio institucional fue arduo y se llevó acabo en cuatro etapas: recopilación y selección del material, capacitación y restructuración del área, digitalización e implementación técnica-tecnológica y en la cual intervinieron 20 personas de siete áreas.

La finalidad del repositorio institucional es la preservación y la gestión al acceso electrónico y contenidos de calidad que se producen en el Instituto y cuyo beneficio es difundir los resultados de la investigación, el acceso a los documentos, promover una mayor consulta, aumentar el nivel de citación de los investigadores del instituto, incrementar la visibilidad y aumentar el impacto de la producción científica y conservar y preservar la producción intelectual de los médicos, trabajadores de la Salud e investigadores del INP.

El repositorio institucional está organizado por comunidades: Acta Pediátrica de México, artículos, artículos INP, artículos de investigadores y tesis; hasta ahora se tiene digitalizados 250 artículos, de 1097 investigadores, se cuentan con 1243 tesis del 2000 al 2015 y se está integrando el 2016 y 2017. El acceso es a través de la página web del Instituto www.pediatria.gob.mx en la pestaña de Enseñanza hay un sublink al Centro de Información y Documentación Científica Biblioteca digital - en donde se encuentra el link del repositorio institucional. La forma de consultarlo algún documento es por nombre o apellido del autor, tema o año de publicación.

El INP se sitúa como la primera Institución Nacional en contar con una plataforma de esta magnitud, en formato digital que facilitará el acceso de la comunidad científica nacional e internacional a la producción científica y académica y en la que el acceso puede hacerse desde cualquier parte del mundo, desde cualquier dispositivo (tableta, computadora o celular) y puede descargarse para su citación o bien contribuir a acrecentar la veta de investigación o tomar otros derroteros. Los invitamos a nutrir esta plataforma, a difundirla y consultarla, lo que contribuirá a la investigación médica a favor de la niñez de México.

Alejandro Serrano-Sierra

Director General

José Nicolás Reynés-Manzur

Director de Enseñanza

reynes@unam.mx

Instituto Nacional de Pediatría 